\title{
Case Report-An unusual diagnosis of a gastric phytobezoar in a middle aged woman.
}

\author{
Rumbidzai Chineka ${ }^{1}$, Tongesai Mukosera ${ }^{1}$, Narcisious Dzvanga ${ }^{1}$, and Brian Gwanda ${ }^{1}$ \\ ${ }^{1}$ United Bulawayo Hospitals
}

February 3, 2021

\begin{abstract}
A phytobezoar occurring in the stomach is uncommon. The most frequent symptoms are dyspepsia, abdominal pain, nausea and vomiting. It is an important differential to remember in those patients with risk factors for developing phytobezoars, as phytobezoars can be managed conservatively in most cases.
\end{abstract}

Case Report-An unusual diagnosis of a gastric phytobezoar in a middle aged woman

\section{Key Clinical Message}

A gastric phytobezoar is uncommon. The most frequent symptoms are dyspepsia, abdominal pain, nausea and vomiting1. It is an important differential to remember in those patients with risk factors for developing phytobezoars, as phytobezoars can be managed conservatively in most cases.

\section{Introduction}

A phytobezoar is a bezoar or trapped mass in the gastrointestinal system that consists of indigestible plant material such as fibers, skins and seeds 1.Most phytobezoars occur in patients who have impaired gastric motility or digestion, usually following gastric surgery(such as Billroth 1 or 2 gastrectomy)or as a consequence of impaired motility in patients with diabetic gastroparesis, mixed connective tissue disease or hypothyroidism. Impaired gastric peristalsis, low gastric acidity,peptic ulcer disease,chronic gastritis and loss of normal pyloric function can all contribute to phytobezoar formation 2,7.Gastric bezoars are usually asymptomatic however when symptoms are present,the most common include postprandial fullness, abdominal pain, nausea, vomiting, anorexia and weight loss 3 .We are presenting the case of a gastric phytobezoar in diabetic middle aged female patient .

\section{Case}

We are presenting the case of a 44 year old female patient who presented with a 6 week history of a productive cough of white sputum ,she denied having any night sweats or fever and she did not have hemoptysis. She had loss of weight, poor appetite as she was vomiting intermittently and had persistent epigastric pain that was not responding to analgesics and which had worsened a few days prior to admission. The pain was aggravated by eating and lasting for a few minutes to hours.In her past medical history the patient had been diabetic for 19 years and was being managed with Actraphane 25 units subcutaneously twice daily. On admission she had no record of how well controlled her diabetes had been over the years. She had also been treated for sputum positive pulmonary Tuberculosis 9 years prior to admission. The patient also had a history of being managed for chronic gastritis with triple ulcer therapy after she had an Oesophageal Gastro-Duodenoscopy(OGD) and subsequent histology was positive for Helicobacter Pylori. 
On examination she was cachetic,had mild pallor,she was apyrexial with a temperature of 36.6, her blood pressure was slightly elevated at $140 / 90 \mathrm{mmHg}$ and she had a regular full volume pulse of $80 \mathrm{bpm}$. Her blood sugar level was elevated at $11.7 \mathrm{mmol} / 1$.She was not in respiratory distress but she did have dull percussion note in the lower zone of the left lung. On examination of the abdomen she had epigastric tenderness however there were no palpable abdominal masses.Her laboratory investigations were as follows Full blood count WCC11.6 $\mathrm{Hb}$ 11.6 MCV 80 and Platelets 322 Urea and Electrolytes were normal with an EGFR of 85 ,creatinine of 84 ,potassium of 3.8 ,sodium of 131 ,urea of 6.1 and bicarbonate of 28 .Her HBA1c was $9.6 \%$ indicating poor glycemic control.Serum IgG for Helicobacter pylori was negative.Her CXRAY showed tracheal deviation to the left,with reduced lung volume of the left lung and compensatory emphysema of the right lung suggestive of fibrotic changes secondary to old Tuberculosis. There were also cavities in the left apex and upper lobe suggestive of current Tuberculosis infection this was confirmed by a positive Ziehl-Neelson(ZN) stain in her sputum.

The patient was admitted into hospital and commenced on TB treatment,her diabetic management was optimized.She was also given Omperazole $20 \mathrm{mg}$ po twice daily for her symptoms of gastritis and some Paracetamol $1 \mathrm{~g}$ po four times daily as analgesia. The patient however continued to vomit and was still complaining of abdominal pain. Serum amylase and lipase levels were ordered both of which were normal. Her abdominal ultrasound scan was also unremarkable. A barium meal and follow through showed a normal stomach outline with slow gastric emptying. There were no strictures or tumors neither were there any filling defects. She then had an Oesophageal Gastro Duodenoscopy(OGD) which showed a mass in the body of the stomach along the greater curvature. There was lots of food debris and vegetable matter seen. The histology for biopsies taken during the OGD reported severe chronic active gastritis and heavy growth of Helicobacter Pylori organisms in the surface mucus. There was no intestinal metaplasia or epithelial dysplasia. An abdominal CT scan was unremarkable and there were no gastric or extragastric masses demonstrated. At this point we added the possibility of phytobezoar in the stomach to our list of differentials. The patient underwent gastric lavage with normal saline. A repeat OGD soon after gastric lavage revealed smooth stomach mucosa and the absence of any mass. The patient's abdominal symptoms improved significantly and there were no residual symptoms of mechanical obstruction in the lower gastrointestinal system. She was discharged home on TB medication, Helicobacter Pylori eradication medication and her usual insulin regimen. She has since been followed up regularly and is doing very well. 


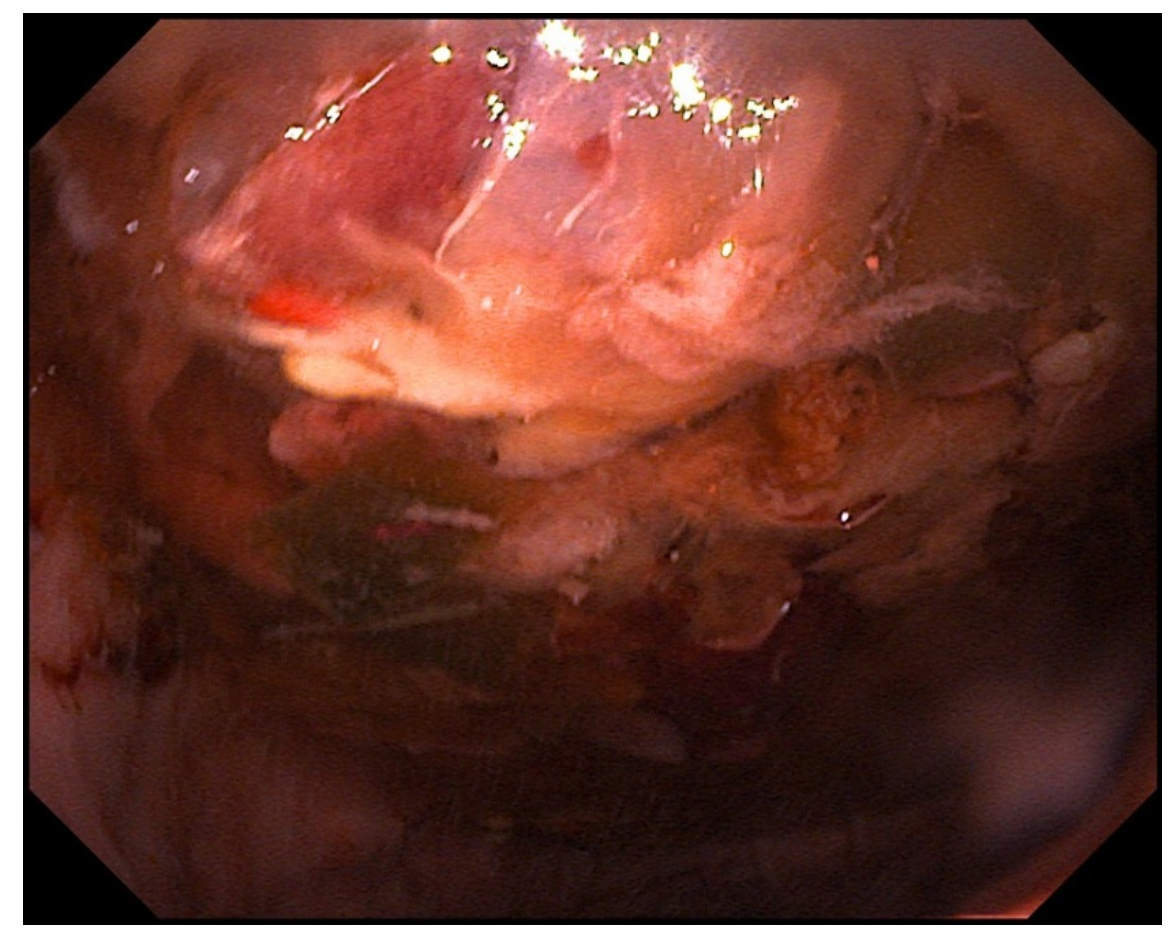

Figure 1 An OGD picture which showed a mass in the body of the stomach along the greater curvature. There was lots of food debris and vegetable matter seen.

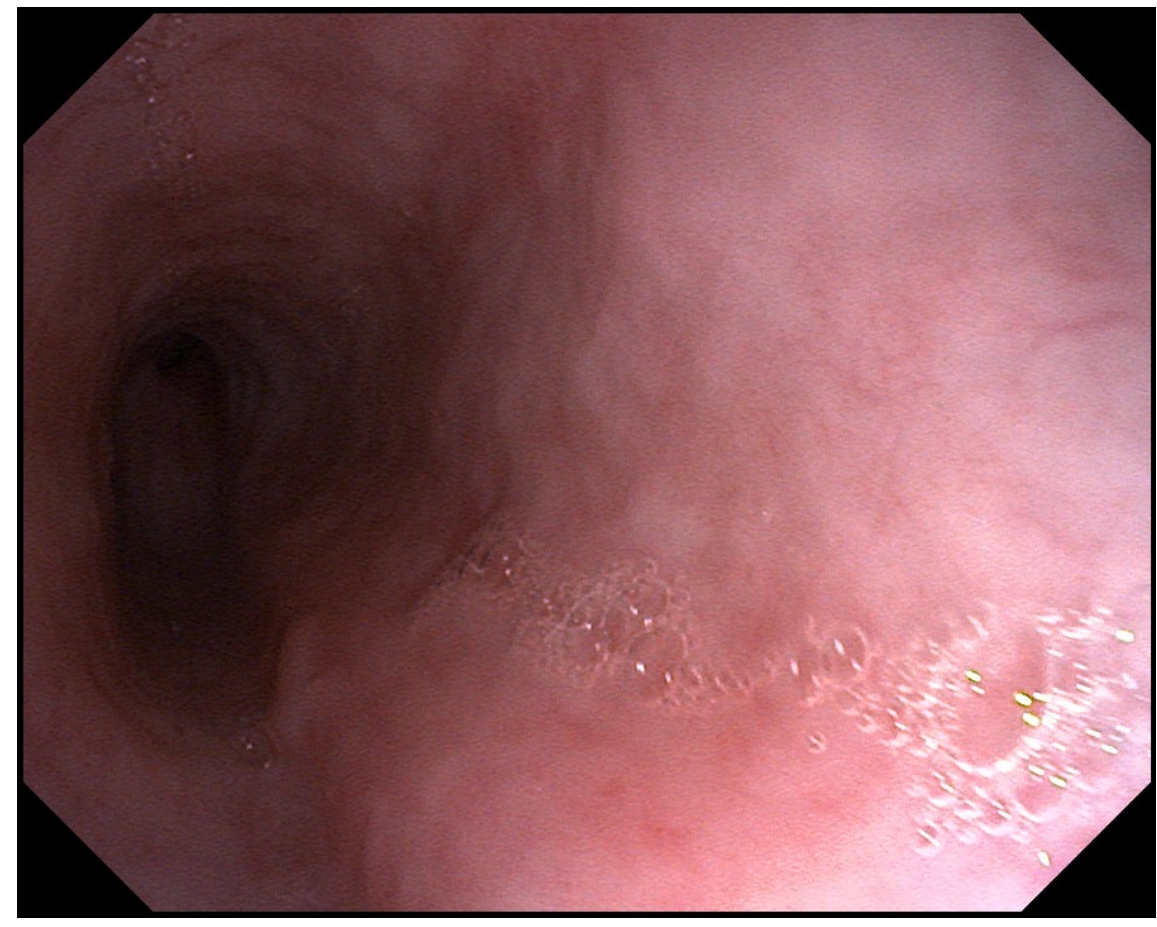

Figure 2 An OGD image soon after gastric lavage revealed smooth stomach mucosa and the absence of any mass. 


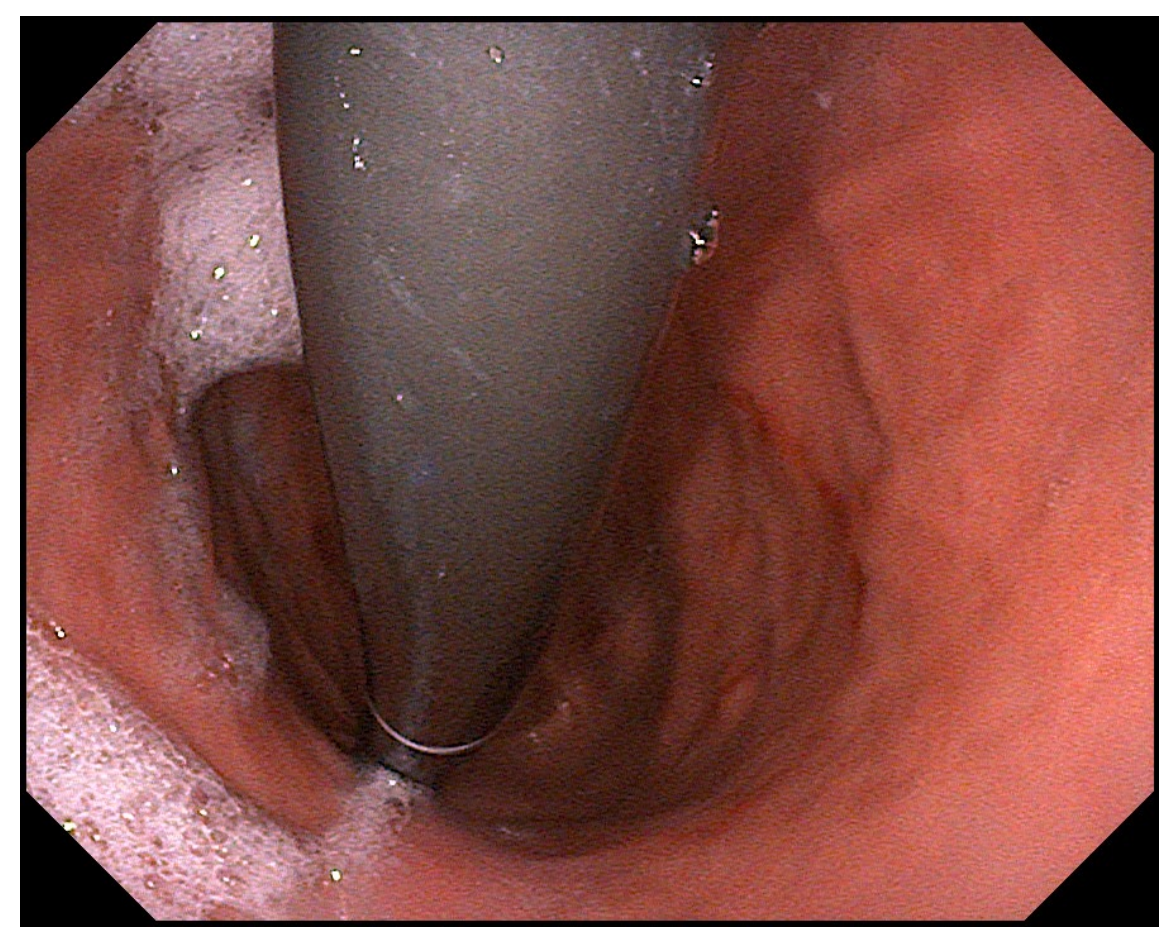

Figure 3 This is an OGD image showing the retroflexed scope or $\mathrm{J}$ manoeuvre to be sure that the mass is no longer present

\section{Discussion}

A bezoar is an indigestible mass of material such as hair, food, seeds or another ingested substance found in the gastrointestinal tract 1.Bezoars are classified into four types according to their material makeup:phtyobezoars, made of vegetables and fibers, trichobezoars, resulting from ingestion of hair and frequently an expression of psychiatric disorders, pharmacobezoars, caused by drugs and medication and lactobezoars which are formed of milk curd 4. Their incidence has been reported to be about $0.4 \% 5$.Phytobezoars are the most common type of bezoars and the most common risk factors for phytobezoar formation are previous gastric surgery, poor mastication, overindulgence of food with high fiber content, diabetes mellitus complicated by gastroparesis and coexisting diseases with delayed gastric emptying such as mixed connective tissue diseases and hypothyroidism 6.Impaired gastric peristalsis, low gastric acidity, and loss of normal pyloric function can all contribute to phytobezoar formation 1.Patients with phytobezoars may experience epigastric pain or discomfort, nausea, vomiting, early satiety, weight loss, diarrhea, dysphagia or upper gastrointestinal ulcerations and hemorrhage 1.6.

Endoscopic examinations play the most important role in the detection of gastric bezoars as well as in the treatment of this disease. A phytobezoar is typically observed in the gastric fundus as a single mass but it can be multiple. The colour is diverse depending on the materials constituting the phytobezoar, ranging from beige, tan, ocher, yellow green, to black. CT scanning is useful to detect both gastric and small intestinal bezoars. Phytobezoars are visualized by CT scans as an ovoid or round occupational mass in the gastrointestinal tract with air bubbles retained inside and a mottled appearance 4,7,8.

Prior to the 1960s , phytobezoars were often treated with surgery, however presently most treatment is conservative.1,4.A wide variety of therapeutic options have since been reported, including medical treatment with cellulase, papain, metoclopramide or N-acetylcysteine 1.Chemical dissolution is another treatment option using either Coca-cola lavages or hydrolytic solutions 4.In cases that are urgent such as those manifesting gastrointestinal bleeding and or ileus, and patients with refractory bezoars, endoscopic fragmentation or 
surgical removal should be applied. Spontaneous disappearance of a bezoar without any specific treatment has also been observed in some patients, however careful follow-up is required in these patients and should only be applied if they are in a stable condition. 8

\section{Conclusion}

The case presented in this article is interesting because the diagnosis of a phytobezoar was unexpected even though our patient was diabetic and this is a known risk factor. Early and accurate diagnosis of phytobezoars is important as conservative management is a safe and effective method of treatment.Furthermore endoscopic saline irrigation was used successfully in the management of this case which is a method not widely described in literature. The multidisciplinary team of Physicians and Surgeons averted unnecessary exlploratory laparotomy.

\section{Authorship}

Author 1 Dr Rumbidzai Chineka, $\mathrm{MBChB}(\mathrm{UZ}), \mathrm{FCP}(\mathrm{SA})$ Senior registrar in General Internal Medicine United Bulawayo Hospitals.Author of the case report and part of the team that managed the patient.

Author 2 Mr Tongesai Mukosera,MBChB(UZ),FCS(COSESCA)Consultant General Surgeon United Bulawayo Hospitals.Author of the case report,performed the diagnostic oesophageal-duodenoscopy and managed the patient.

\section{Acknowledgements}

Dr Narcisious Dzvanga MMed (UZ), FCP (ECSACOP) Consultant Physician. Physician in charge of patient, review of case report and guidance in writing the case.

Mr Brian Gwanda MBChB(UZ),FCS(COSESCA), Consultant General Surgeon.Part of the surgical team that managed the patient.

\section{References}

1. Fengbo Tan,Hongbin Mo,Xiao He,Haiping Pei,An unsual case of gastric outlet obstruction caused by giant persimmon phytobezoars,Gastroenterology Report,Volume 7,Isuue 1 ,February 2019,Pages 74-76.

2. Scott J. Kramer,Mark B. Prochapin,Gastric Phytobezoar Dissolution with ingestion of diet coke and cellulase,Gastroenterol Hepatol (N Y).2012 Nov;8(11):770-772.

3. O.S.Balogun,A.O. Osinowo,M.O.Afolayan, and A.A. Adesanya,An obstructing small bowel phytobezoar in an elderly female Nigerian:A case report and literature review,Case reports in Surgery,Case report,Open access, Volume 2017.

4. S.Occhionorelli,M.Zese,G.Vasquez,A rare case of a double phytobezoar causing gastric and jejunum obstruction in an adult man: a case report,Journal of Medical Case Reports 10,Article number:350(2016).

5. S.D.Ladas,D.Kamberoglou,G.Karamanolis,J.Vlachogiannakos,I Zouboulis-Vafiadis,Systemic review:Coca-cola can effectively dissolve gastric phytobezoars as a first line treatment,Alimentary Pharmacology and Theraeutics, Volume 37,Issue 2.

6. Ronald L. Eisenberg,Marc S.Levine,Miscellaneous Abnormalities of the stomach and duodenum,Textbook of Gastrointestinal radiology $3^{\text {rd }}$ edition,2008.

7. Masaya Iwamuro,Hiroyuki Okada,Kazuhide Yamamoto,review of the diagnosis and management of gastrointestinal bezoars,World J Gastrointest Endosc.2015 Apr 16;7(4):336-345.

8. Benjamin R Kuhn,Adam G. Mezoff,Bezoars,Pediatric Gastrointestinal and Liver Disease $4^{\text {th }}$ edition,2011. 

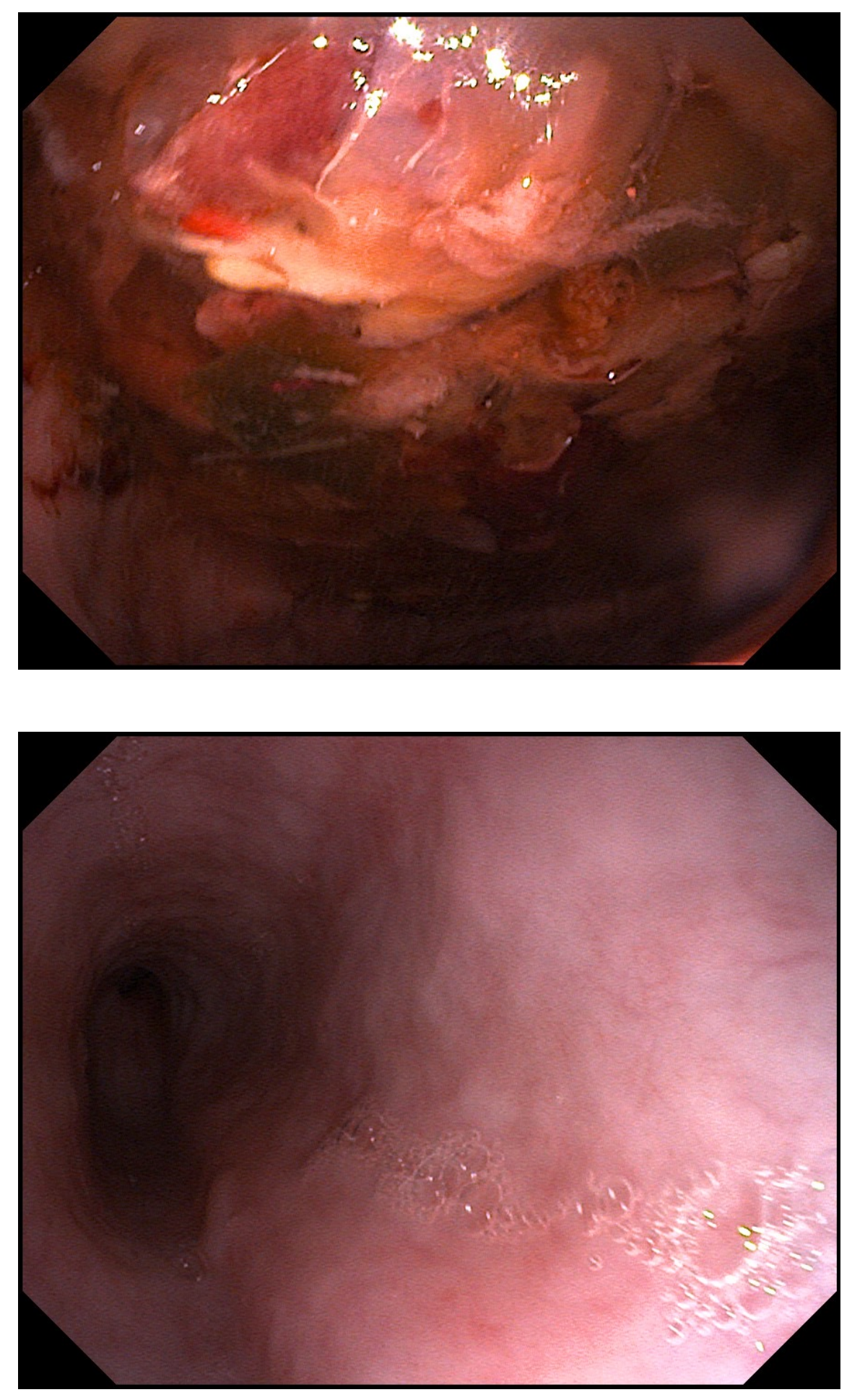


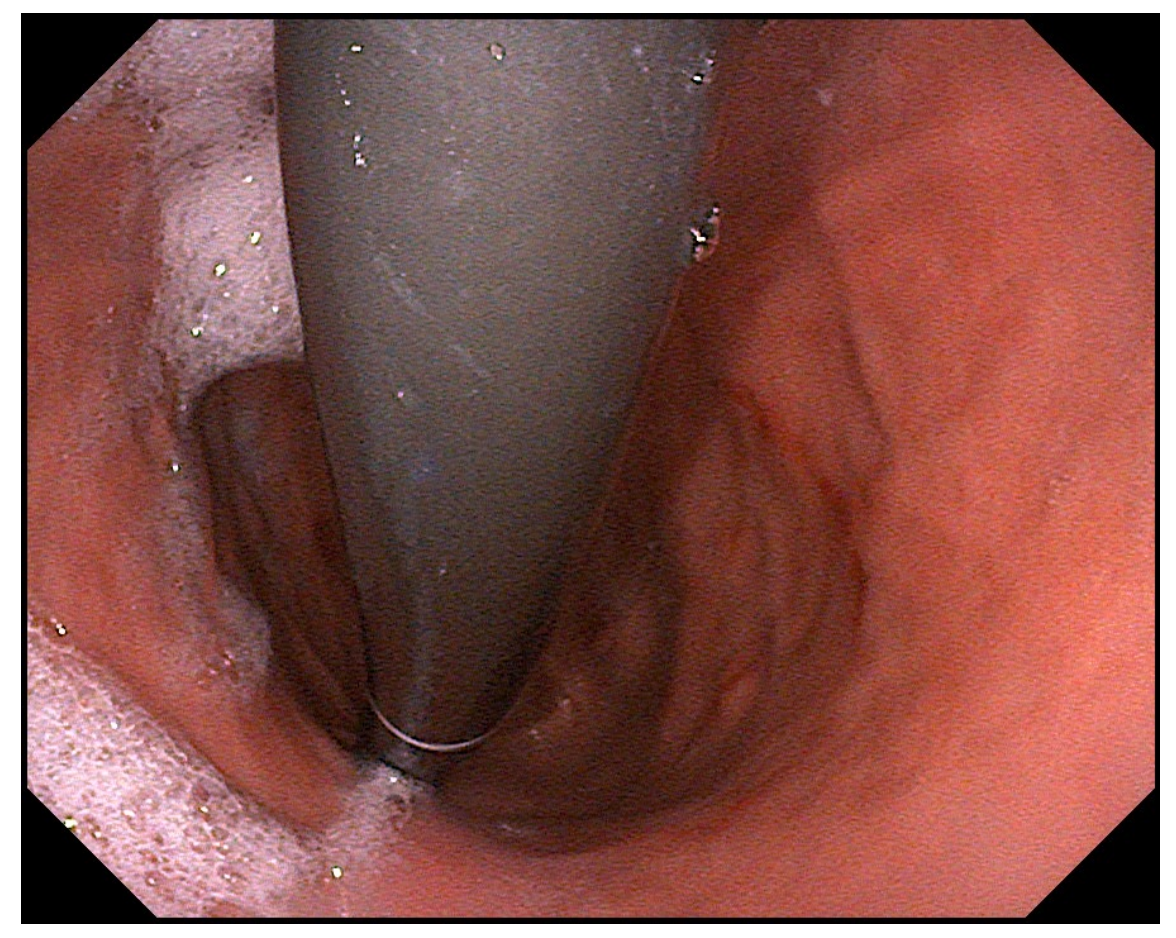

